\title{
Seasonality of Rotavirus Infection with its Vaccination
}

\author{
E. Shim, H.T. Banks, and C. Castillo-Chavez
}

\begin{abstract}
The impact of rotavirus, the most prevalent diarrheal pathogen in young children worldwide, may be reduced by either a recently approved vaccine or others in development. The dynamics of rotavirus infections are studied using a simple mathematical model that includes the impact of breast feeding, seasonality and the possibility of control via vaccination. Data from Australia are fitted to a model that incorporates the effect of seasonality in the transmission process. The impact of temporary and partially effective vaccines is explored. Finally, the issue of cost is briefly discussed in the context of identifying "optimal" vaccination strategies.
\end{abstract}

\section{On the characteristics of rotavirus}

Rotavirus spreads easily especially among infants. In fact, $95 \%$ of children worldwide are infected by the time they reach age 5 with peak incidence occurring between ages 4 months and 36 months [34]. CDC epidemiologist Umesh Parashar reported at an international meeting on the epidemiology of the rotavirus held in Mexico City (2004) that rotavirus was detected in $60 \%$ of stool samples from children hospitalized with severe diarrhea in Vietnam; $41 \%$ in China; $56 \%$ in Myanmar; and $29 \%$ in Hong Kong ([36] and [37]). In the United States, approximately 2.7 million children under 5 years of age are infected by rotavirus each year, resulting in 500,000 visits to physicians and 50,000 hospitalizations per year. The estimated direct cost of medical care is about $\$ 274$ million per year in the U.S. alone ([35] and [44]). In Peru, for example, the total medical care costs directly linked to rotavirus infections are estimated to be around $\$ 2.6$ million (U.S.) a year [55], an amount that includes the costs associated with 64,000 clinic visits and 30,000 hospitalizations per year. A study presented at the European Society for the Study and Prevention of Infant Death (ESPID) quantified the burden associated with rotavirus disease in the new European Union (EU). Their estimates used a model recently developed by the U.S. Centers for Disease Control and Prevention (CDC). The EU managed

1991 Mathematics Subject Classification. Primary 92D30; Secondary 62 F25.

Key words and phrases. rotavirus; vaccine; Rotarix; RotaTeq; seasonal

This research was partially done during the visit of E. Shim and Dr. C. Castillo-Chavez to the Statistical and Applied Mathematical Science Institute (SAMSI), Research Triangle Park, NC, which is funded by NSF under grant DMS-0112069, and to the Center for Research in Scientific Computation at N.C. State University. This research was supported in part by the Joint DMS/NIGMS Initiative to Support Research in the Area of Mathematical Biology under grant 1R01GM67299-01(Dr. H.T.Banks) and by NSA grant H98230-05-1-0097 (Dr. C. CastilloChavez) as well as NSF grant DMS-05-2349 (Dr. C. Castillo-Chavez). 
to obtain country specific estimates of rotavirus levels of infections in each of its 25 member states ([46] and [50]). These estimates revealed nearly 4.5 million episodes of rotaviral disease among the 24 million children under 5 years in the EU ([46] and $[50])$.

The dynamics of rotavirus infections are not "straight forward". In fact, geographically driven seasonal patterns seem to be prevalent in some regions of world. In moderate climates, rotavirus diarrhea occurs predominantly during the fall and winter. Peak rotavirus activity in the United States begins in the Southwest in autumn (October through December) with an epidemic "wave" that ends in the Northeast in the spring (March through May) [42]. Although the reason for this pattern is unknown, a high correlation between latitude and the timing / magnitude of rotavirus infection outbreaks have been identified [12]. Seasonality is less marked in tropical settings and in developing countries [12]. The predominant mode of rotavirus transmission is fecal-oral [34]. It spreads through respiratory secretions and person-to-person contact. The incubation period for rotavirus disease is approximately 2 days and its symptoms including vomiting and watery diarrhea usually last for 3 - 8 days. Although immunity after infection is temporary, subsequent infections tend to be less severe than the original infection. Contaminated environmental surfaces have also been identified as possible mechanism for infections but high rates of infections among infants younger than three years old have been identified regardless of sanitary conditions [34]. That is, rotavirus transmission rates seem unaffected by improvements in sanitation and hygiene. The cost of treatment is an issue as many countries in the developing world (575 million children under age five) often do not have the resources to provide life-saving intravenous treatment to rehydrate children with severe rotavirus diarrhea [54].

Rotavirus has seven major groups (A-G) while most human strains belong to group A [34]. Of the group A serotypes, at least ten $\mathrm{G}$ and eight $\mathrm{P}$ serotypes of human rotavirus have been characterized [34]. Among 80 theoretically possible different strains of rotavirus four common strains (P[8]G1; P[8]G3; P[8]G4; and $\mathrm{P}[4] \mathrm{G} 2)$ dominate globally [34].

In Section 2 we introduce a simple model that includes infants protected by maternal antibodies, vaccinated children (after effective breast-feeding ends), and seasonality in transmission rates. In the absence of seasonality, the basic reproductive ratio is computed and interpreted. Section 3 reports on fitting a vaccine-free model with seasonal transmission to the data and exploration of the role of vaccination. In order to consider the impact of cost in the implementation of a vaccine program in Section 4 we briefly review what is known about the implementation and development of rotavirus vaccine. In Section 5 the model of Section 3 is expanded to include the possibility of various vaccine schemes. In Section 6 , a concept of optimal vaccination is reviewed in the context of an age-structured population and a brief summary of prior recent results $([\mathbf{4 0}])$ is presented. Finally in Section 7 we revisit the results presented here and outline possible future directions of research.

\section{Model with seasonality and the role of maternal antibodies}

A simple model is introduced in order to test the role of maternal antibodies both theoretically and through simulations. The model (see Figure 1) includes breast-feeding $(M(t))$, susceptible $(S(t))$, latently infected $(L(t))$, infectious $(I(t))$ and temporarily recovered $(R(t))$ classes. It is assumed that the total population, 
$N=M+S+L+I+R$, is constant. This is appropriate since demographic changes and rotavirus-induced mortality are negligible under the temporal scale consideration.

Although there are several studies supporting the role of maternal antibodies in protecting infants against rotavirus infections there are some conflicting data concerning this issue [31]. Several studies indicate that breast-feeding protects against symptomatic rotavirus infection $([\mathbf{1 1}],[\mathbf{2 8}]$ and $[\mathbf{2 9}])$. Also some state that breast-feeding reduces the severity of symptoms although rotavirus infection occurs in breast-fed children $([\mathbf{3}],[\mathbf{1 1}],[\mathbf{1 6}]$ and $[\mathbf{1 8}])$. Others conclude that breast-feeding has a protective effect especially among newborns ([4], [9], [14], $[\mathbf{2 2}]$ and $[\mathbf{4 3}])$. Furthermore it is shown that rotavirus infections are uncommon in the first months of life among intensively breast-fed infants and that infection rates increase with age and reach maximal values around the six to eighteen month age period $[\mathbf{2 7}]$. In this paper we assume the protectional role of maternal antibodies provided by breast-feeding. Therefore the infection rate among infants who are breast-fed is assumed to be reduced by factor $\xi$ where $0<\xi<1$ over a short window in time. In fact, it is assumed that only the proportion $q$ of newborns per unit time $(\mu N)$ is breast-fed with an average effective immune period to rotavirus infection (due to breast-feeding) of $1 / \rho$. The average life span of an individual is $1 / \mu$. The total death rate $(\mu(M+S+L+I+R))$ is assumed to be equal to total birth rate $(\mu N)$; $1 /(\alpha+\mu)$ is the average effective latent period; $1 /(\gamma+\mu)$ is the average effective infectious period; $\beta$ is the transmission rate per infective; and $I / N$ is the proportion of contacts between susceptibles and infectives, that is, random mixing is assumed. Finally $\beta$ is assumed to depend on the season and consequently, it is modeled by $\beta(t)=\beta_{0}\left[1+\beta_{1} \cos (2 \pi(t-\tau))\right]$ where $\beta_{0}$ gives the mean transmission rate, $\beta_{1}$ its amplitude and $\tau$ the lag-associated with seasonal transmission. Using these definitions, assumptions and Figure 1 we arrive at the following non-autonomous nonlinear system that models the transmission dynamics of rotavirus infections in a homogeneously mixing population:

$$
\begin{aligned}
& M^{\prime}=q \mu N-\xi \beta M I / N-(\rho+\mu) M, \\
& S^{\prime}=\mu[(1-q) N-S]+\rho M-\beta S I / N+\delta R, \\
& L^{\prime}=\xi \beta M I / N+\beta S I / N-(\alpha+\mu) L, \\
& I^{\prime}=\alpha L-(\mu+\gamma) I, \\
& R^{\prime}=\gamma I-(\mu+\delta) R,
\end{aligned}
$$

where $N=M+S+L+I+R$ and $\beta=\beta_{0}\left[1+\beta_{1} \cos (2 \pi(t-\tau))\right]$.

When $\beta=\beta_{0}$ and $\beta_{1} \equiv 0$, that is, in the absence of seasonality, the reproductive number can be easily computed. We get

$$
\Re(q, \rho)=\frac{\alpha \beta_{0}[\rho+\mu(1-q(1-\xi))]}{(\rho+\mu)(\alpha+\mu)(\gamma+\mu)} .
$$

with the basic reproductive ratio being given $\Re_{0}=\Re(0,0)$. $\Re_{0}$ is the number of secondary infections generated by a typical infectious individual in a population of primarily susceptible individuals. Since our model considers breast feeding as a "control" mechanism then

$$
\Re_{0}=\frac{\beta_{0}}{\gamma+\mu} \times \frac{\alpha}{\alpha+\mu}
$$




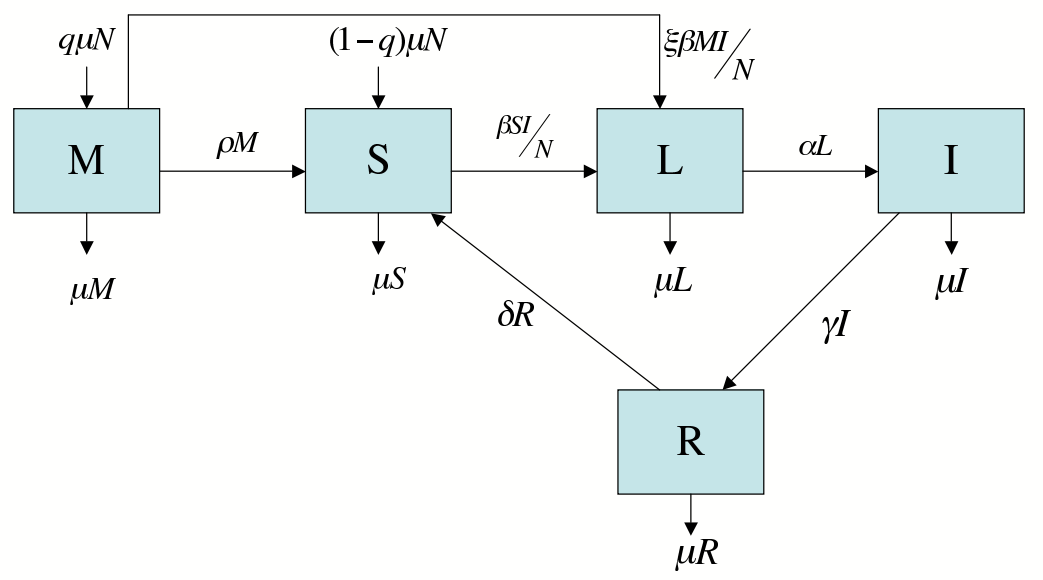

Figure 1. A schematic representation of the flow of individuals between epidemiological classes for System (2.1).

That is, $\Re_{0}$ is the product of the (non-seasonal) transmission rate, the effective infectious period $\left(\frac{1}{\gamma+\mu}\right)$ and the proportion of latently infected $\left(\frac{\alpha}{\alpha+\mu}\right)$ individuals progressing towards the infectious stage. Breast-feeding, as modeled by $q$, plays the role of a "temporarily effective vaccine" given at birth to the proportion $q$ of newborns (a model involving neonatal vaccines under development is given in Section 5). It can be shown that if $\Re(q, \rho)<1$ then the disease-free equilibrium is stable while if $\Re(q, \rho)>1$ then it is unstable. Consequently, rotavirus infections disappear if $\Re(q, \rho)<1$ but persist when $\Re(q, \rho)>1$. We observe that although breast feeding is unlikely to control the disease (current situation), it is capable of reducing rotavirus' disease prevalence (long-term dynamics).

With reasonable and best-fit parameters (from Section 3) we can get a rough estimation of the current replacement ratio calculated at oscillatory endemic steady state as 1.0348. A value near one would be expected since the disease is "endemic". That is, a replacement number around 1 should be in operation. Nevertheless it is "surprising" to arrive to such a close estimate via a general estimation procedure.

Ideally, the estimation of $\Re_{0}$ would require rotavirus invasion data on a population where prior rotavirus infections and breast-feeding have not been experienced. Fortunately some rough alternatives are available. Theoreticians have arrived at various estimates. The simplest is given by

$$
\Re_{0}=1+\frac{W}{A}
$$


where $A$ is the mean age at first-infection and $W$ is the average "life-span" of an individual. Formulae that include more detailed population characteristics have been computed in [5].

From the study conducted in [45] which focuses on the reinfections of infants, we gathered the following data: $67 \%$ of children become infected with rotavirus by age one and $96 \%$ by age two. Assuming that most infections in children are generated by other children (humans may play the role of "vectors"), we can roughly conclude that the average time spent ("life-span") by children in environments where there is a high risk of infections is only a few years (possibly three). The above data suggest that the average age of the first infection is less than one. Consequently Eq. (2.3) roughly suggests that $2<\Re_{0}<5$ in a population of infants.

The rotavirus-infection population data used in this manuscript are from Australia. The data do not come from a census but rather from a sample and extrapolation [6]. An approximate "representative" sample of rotavirus-positive specimens was collected and the sample data extrapolated to generate a national scale data [6]. The seasonal peaks in Victoria (eastern Australia) generally occurred regularly in the months of July to September. Researchers used a regression model on the monthly incidence of hospital admission for acute gastroenteritis in children under five years of age in Victoria during the period 1993-96. Their model made the following assumptions: (i) rotavirus is the only major cause of childhood gastroenteritis admissions that exhibits significant seasonal variation; and (ii) the total number of hospital admissions due to rotavirus in Victoria is a constant multiple of the number recorded in sample hospitals. Thus, total admissions per month were expressed as the sum of a constant number (representing non-rotavirus causes) and varying numbers of rotavirus admissions. The resulting linear regression model for the number of admissions per month in selective Australian states was used to estimate the proportion of hospital admissions for acute gastroenteritis resulting from rotavirus infections in Victoria.

In order to better understand their results, we describe their regression model [6]. $N_{i}$ denotes the number of total acute gastroenteritis admissions in Victoria in month $i ; n_{i}$ the number of "rotavirus-positive" admissions in the participating hospital(s) in month $i ; \zeta$ the monthly number of admissions in Victoria not caused by rotavirus; $\eta$ a scaling factor relating the number of "rotavirus-positive" admissions in the whole State of Victoria to that in the sample of hospitals; and $\varepsilon_{i}$ is used to denote random measurement error [6]. Their regression model is

$$
N_{i}=\zeta+\eta n_{i}+\varepsilon_{i}
$$

Carlin et al. ([6]) used ordinary least squares to estimate $\eta$ on the understanding that its value would be underestimated by this model due to the lack of perfect correlation between rotavirus incidence recorded at index hospitals and total rotavirus infections (at all hospitals) in Victoria.

Although we have used the rescaled data in [6], we believe that since most infections take place among infants under five years old a weighted re-scaling that heavily favors impact on younger children was probably more appropriate. However, our goal here is not to make specific predictions but rather to illustrate the role of epidemiological models in the evaluation of the relative impact of various vaccine regimes. 


\section{Parameter Estimation}

In this section we fit the model solution to data from Victoria in Australia. The data set is limited and the number of model parameters large. However the goal is to estimate only some of the parameters that best fit the data to gain additional insights on the epidemic process. Some parameter estimates will be used as "baseline" model estimates and used to conduct a preliminary study on the potential impact of a rotavirus vaccine on disease prevalence.

The model parameters $\theta=\left(\rho, \beta_{0}, \beta_{1}, \delta, \gamma, \tau\right)\left(\theta \in \Re^{p}\right.$ with $p=6$ in this case $)$ are fitted to the monthly incidence number of rotavirus cases in Victoria $([\mathbf{6}])$ via a least-square fit of model solutions. We use the computer program (MATLAB, The MathWorks, Inc.) using initial conditions for the parameters in the intervals: $0.002<\rho<0.2$ (about $97 \%$ of all breast-feeding duration in Australia fall in this range [53]), $0<\beta_{0}<1,0<\beta_{1}<1$ (so that force of infection is always nonnegative), $0<\delta<1,130<\tau<150$ (to consider seasonal pattern in Victoria), $0.1<\gamma<0.5$ (recovery period is known as approximately five days indicating $\gamma \approx 0.2$ ). Multiple fitting is carried out using selected values of $\xi$, all around 0.4 as this seems to be an accepted value, that is, $40 \%$ protection is conferred by breastfeeding during the first year of life [36]. There also seems to be strong evidence that about $50 \%$ of breast-fed babies under six months are fully-protected from rotavirus infections [36].

Our $n$ scalar longitudinal observations (time series of number of infective individuals) are

$$
I_{j} \equiv f\left(t_{j}, \theta_{0}\right)+\epsilon_{j}, j=1,2, \ldots n,
$$

where $f\left(t_{j}, \theta_{0}\right)$ is a numerical solution of the fourth equation in System (2.1) and $\theta_{0}$ is a "set" of theoretical "true" parameter values (assumed to exist in a standard statistical approach). We assume for our statistical model of the observation or measurement process (3.1) that the errors $\epsilon_{j}, j=1,2, \ldots, n$, are independent identically distributed (i.i.d.) random variables with mean $E\left[\epsilon_{j}\right]=0$ and constant variance $\operatorname{var}\left[\epsilon_{j}\right]=\sigma_{0}^{2}$ where of course $\sigma_{0}^{2}$ is unknown. We then have that the observations $I_{j}$ are $i . i . d$. with mean $E\left[I_{j}\right]=f\left(t_{j}, \theta_{0}\right)$ and variance $\operatorname{var}\left[I_{j}\right]=\sigma_{0}^{2}$.

We estimate parameters using an OLS (ordinary least squares) approach. Thus we seek to use the observations $\left\{I_{j}\right\}$ with the model to seek $\hat{\theta}$ that minimizes

$$
J(\theta)=\sum_{j=1}^{n}\left|I_{j}-f\left(t_{j}, \theta\right)\right|^{2} .
$$

Since $I_{j}$ is a random variable, we have that the estimator $\hat{\theta}_{O L S}$ is also a random variable with a distribution called the sampling distribution. Knowledge of this sampling distribution provides uncertainty information (e.g., standard errors) for the numerical values of $\hat{\theta}$ obtained using a specific data set (i.e., a realization of $\left\{I_{j}\right\}$ ) when minimizing $J(\theta)$.

Under reasonable assumptions on smoothness and regularity (the smoothness requirements for model solutions are readily verified for our example; the regularity requirements involve, among others, conditions on how the observations are taken as sample size increases, i.e., $n \rightarrow \infty)$, the standard nonlinear regression approximation theory $([\mathbf{1 5}],[\mathbf{1 7}],[\mathbf{2 3}]$, and Chapter 12 of $[\mathbf{3 9}])$ for asymptotic (as $n \rightarrow \infty)$ distributions can be invoked. This theory yields that the sampling distribution $\hat{\theta}(Y)$ 
for the estimate $\hat{\theta}$, where $Y=\left\{I_{j}\right\}_{j=1}^{n}$, is approximately a $p$-multivariate Gaussian with mean $E[\hat{\theta}(Y)]$ and covariance matrix $\operatorname{cov}[\hat{\theta}(Y)]=\Sigma_{0}=\sigma_{0}^{2}\left[\chi^{T}\left(\theta_{0}\right) \chi\left(\theta_{0}\right)\right]^{-1}$. Here $\chi(\hat{\theta})=F_{\theta}(\theta)$ is the $n \times p$ sensitivity matrix with elements

$$
\chi_{j k}(\theta)=\frac{\partial f\left(t_{j}, \theta\right)}{\partial \theta_{k}} \quad \text { and } \quad F_{\theta}(\theta)=\left[f_{\theta}\left(t_{1}, \theta\right), \ldots, f_{\theta}\left(t_{n}, \theta\right)\right]^{T} .
$$

That is, for $n$ large the sampling distribution approximately satisfies:

$$
\hat{\theta}_{O L S}(Y) \sim \mathcal{N}_{p}\left(\theta_{0}, \sigma_{0}^{2}\left[\chi^{T}\left(\theta_{0}\right) \chi\left(\theta_{0}\right)\right]^{-1}\right):=\mathcal{N}_{p}\left(\theta_{0}, \Sigma_{0}\right) .
$$

The elements of the matrix $\chi=\left(\chi_{j k}\right)$ can be estimated using the forward difference

$$
\chi_{j k}(\theta)=\frac{\partial f\left(t_{j}, \theta\right)}{\partial \theta_{k}} \approx \frac{f\left(t_{j}, \theta+h_{k}\right)-f\left(t_{j}, \theta\right)}{h_{k}},
$$

where $h_{k}$ is a $p$-vector with nonzero entry in only the $k^{\text {th }}$ component, or using sensitivity equations (see $[\mathbf{2}]$ and the references therein). For our efforts here we chose the forward difference approach. Since $\theta_{0}, \sigma_{0}$ are not known, we must approximate them in $\Sigma_{0}=\sigma_{0}^{2}\left[\chi^{T}\left(\theta_{0}\right) \chi\left(\theta_{0}\right)\right]^{-1}$. For this we follow standard practice and use the approximation

$$
\Sigma_{0} \approx \Sigma=\hat{\sigma}^{2}\left[\chi^{T}(\hat{\theta}) \chi(\hat{\theta})\right]^{-1}
$$

where $\hat{\theta}$ is the parameter estimate obtained, and

$$
\sigma_{0}^{2} \approx \hat{\sigma}^{2}=\frac{1}{n-p} \sum_{j=1}^{n}\left(I_{j}-f\left(t_{j}, \hat{\theta}\right)\right)^{2} .
$$

Standard errors to be used in confidence interval calculations are thus given by $S E_{i}=\sqrt{\Sigma_{i i}}, i=1,2, \ldots, p$. In the estimation procedure for $\theta=\left(\rho, \beta_{0}, \beta_{1}, \delta, \gamma, \tau\right)$, we fixed selected parameters to acceptable values: $\alpha=0.5, q=0.876[\mathbf{5 3}]$ and $\mu=3.9139 e-05$. The least square method was implemented and the best estimates for $\theta$ as well as their associated $95 \%$ confidence intervals (see Table 1 and Figure 2) were obtained using the Victoria data. Since we only have data over a four-year period corresponding to $n=49$, we do not pursue further statistical analyses. The parameters obtained are used to test the impact of control measures like vaccinations or increasing breast-feeding numerically given that the disease is endemic. Again, we observe that goal here is not to make specific predictions but rather to explore the impact of various policies within a "realistic" setting.

\section{On the vaccine of rotavirus}

The world's first rotavirus vaccine, RotaShield, was licensed for use in the United States in 1998. From clinical trials conducted in the United States, Finland, and Venezuela, researchers estimated its efficacy to be between 80 and $100 \%$ at preventing severe rotavirus diarrhea $[\mathbf{1 3}]$. No statistically significant serious adverse effects were reported during trials [13].

However in 1999 the U.S. Centers for Disease Control and Prevention (CDC) established an association between RotaShield and a potentially fatal bowel obstruction, called intussusception [37]. This association resulted in the withdrawal of RotaShield from the market in 1999 [25]. The CDC, in cooperation with the Food and Drug Administration (FDA), the National Institutes of Health (NIH), and Public Health Service officials, along with the recommendation of Wyeth-Lederle, 
TABLE 1. Confidence intervals of estimated parameters when $\xi=$ $0.2,0.3,0.4$ and 0.5 respectively

\begin{tabular}{|c||c|c|c|}
\hline parameters & best-fit values & standard error & 95\% confidence intervals \\
\hline$\rho$ & 0.0003 & 0.0000 & {$[0.0003,0.0003]$} \\
& 0.0001 & 0.0000 & {$[0.0001,0.0001]$} \\
& 0.0000 & 0.0000 & {$[0.0000,0.0000]$} \\
& 0.0000 & 0.0000 & {$[0.0000,0.0000]$} \\
\hline$\beta_{0}$ & 0.2077 & 0.0000 & {$[0.2077,0.2077]$} \\
& 0.2073 & 0.0000 & {$[0.2073,0.2073]$} \\
& 0.2099 & 0.0000 & {$[0.2099,0.2099]$} \\
& 0.2070 & 0.0000 & {$[0.2070,0.2070]$} \\
\hline$\beta_{1}$ & 0.2217 & 0.0300 & {$[0.1629,0.2805]$} \\
& 0.2235 & 0.0300 & {$[0.1647,0.2823]$} \\
& 0.2266 & 0.0316 & {$[0.1646,0.2886]$} \\
& 0.2353 & 0.0332 & {$[0.1703,0.3003]$} \\
\hline$\delta$ & 0.1000 & 0.0436 & {$[0.0146,0.1854]$} \\
& 0.1000 & 0.0447 & {$[0.0123,0.1877]$} \\
& 0.1000 & 0.0447 & {$[0.0123,0.1877]$} \\
& 0.1000 & 0.0469 & {$[0.0081,0.1919]$} \\
\hline$\gamma$ & 0.2028 & 0.0000 & {$[0.2028,0.2028]$} \\
& 0.2026 & 0.0000 & {$[0.2026,0.2026]$} \\
& 0.2055 & 0.0000 & {$[0.2055,0.2055]$} \\
& 0.2032 & 0.0000 & {$[0.2032,0.2032]$} \\
\hline$\tau$ & 145.6292 & 3.5942 & {$[138.5846,152.6738]$} \\
& 145.8228 & 3.5768 & {$[138.8123,152.8333]$} \\
& 145.7564 & 3.5411 & {$[138.8159,152.6969]$} \\
& 145.4982 & 3.5180 & {$[138.6030,152.3934]$} \\
\hline
\end{tabular}

presented an expedited review of scientific data on such an association. The advisory Committee on Immunization Practices (ACIP) supported the withdrawal $[51]$.

The cost-benefit analysis within U.S. and the strong association between rhesus rotavirus tetravalent (RRV-TV) vaccination and intussusception did support the withdrawal of RotaShield [32]. However such cost-benefit analyses may lead to different conclusions in countries where children have more than 12 episodes of diarrhea per year and relatively high mortality rates [55]. A rotavirus vaccine in such a setting may save the lives of about 500,000 children annually through its ability to prevent the worst cases of diarrheal disease among infants. In other words, cost-benefit analyses for developed countries may not be applicable elsewhere.

Now we have some new vaccines that are available or under development. The vaccine Rotarix licensed to GlaxoSmithKline (GSK) was introduced in Mexico in 2004. Rotarix is derived from a single strain of human rotavirus (G1, P[8]) [36]. It is a live attenuated vaccine given in two oral doses [36]. GSK conducted clinical trials on over 9,400 subjects during Phase I and II of their clinical trials in the U.S., Latin America, and Europe [36]. Phase III efficacy trials were initiated in the summer of 2003 in Latin America [50]. These Phase III trials involved over 70,000 infants [50]. Rotarix was licensed on July 12th 2004 by the Mexican regulatory 

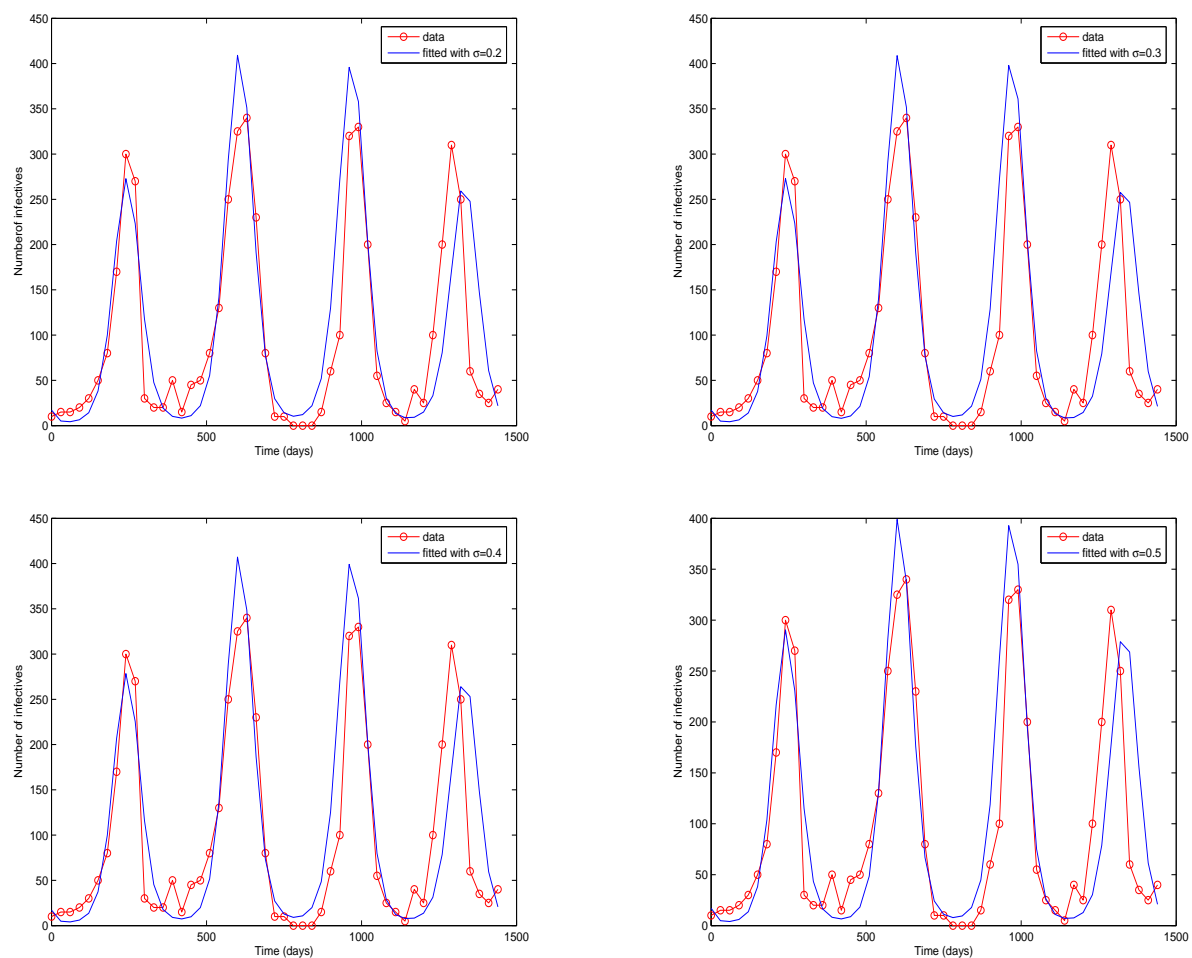

FiguRE 2. Number of rotavirus infected individuals in Victoria, Australia, 1993-1996 ([6]) and the fitted graph in a model with seasonality. Various fixed reductions in infection rate due to breast feeding are used. In fact, $\xi$ values used are $0.2,0.3,0.4$ and 0.5 .

authorities [52]. Applications for approval for Rotarix have been filed in more than 20 countries in Latin America and in Asia [52]. Rotarix is currently under review for approval by the European Agency for the Evaluation of Medicinal Products (EMEA) [50]. It is expected to be introduced in the European Union market in $2006[\mathbf{5 0}]$.

De Vos reported $([\mathbf{3 6}])$ that "Rotarix has about $73 \%$ efficacy against any rotavirus induced gastroenteritis and up to $90 \%$ against severe rotavirus infections". De Vos noted that "the rates of vaccine take (indicated by the strength of the immune response) did vary as a function of the age of the first vaccination" [36]. Rotarix will be given orally, in two doses at 2 and 4 months of age before maternal antibody protection is lost. Rotarix is reported to have a considerably higher incidence of low grade fever as a side effect when compared to RotTeq [47]. There is no diarrhea or vomiting associated with Rotarix and a calcium carbonate buffer in Rotarix protects the attenuated virus from inactivation by stomach acids [47]. A monovalent vaccine approach such as Rotarix is based on the fact that recurrent infections with rotavirus confer immunity against subsequent severe infections [48]. 
It is known that initial antibody response to rotavirus infection is serotype specific while subsequent infections are expected to protect against future infections by other serotypes $([\mathbf{1}],[\mathbf{2 4}]$ and $[\mathbf{4 8}])$.

There is another vaccine candidate. RotaTeq, an attenuated live oral vaccine, which is scheduled to be administered in three doses is also being introduced into the world markets. RotaTeq is a pentavalent bovine-human vaccine that contains five antigens: G1, G2, G3, G4, and P1 [33]. The vaccine is administered orally beginning at age 2 months, with one to two months intervals between doses [33]. The five RotaTeq strains account for over $80 \%$ of rotavirus generated disease worldwide [36]. Clinical trials show that the vaccine efficacy of RotaTeq is approximately $75 \%$ against rotavirus disease, regardless of severity or serotype, and $100 \%$ against severe rotavirus disease [33]. Some researchers found the stool rotavirus IgA response to be an indication of an increased immune response generated by the three doses of RotaTeq [49].

There are some advantages in the use of a multivalent vaccine. Clark and Offit ([10]) note that "Using the analogy with influenza virus, optimum protection against different serotypes may require a vaccine that is precisely homologous in antigen composition and if so, Rotateq would provide protection against the most common serotype PlaG1 because it includes both Pla and G1 rotavirus reassortants". In the same context, RotaTeq is expected to provide superior protection against G2, G3, and G4 wild-type viruses [10], [30]. Clark and Offit ([10]) added that "Confronting a serotype that was not G1, G2, G3, or G4, a Rotateq preparation containing a WC3 reassortant expressing the new G serotype could be formulated readily".

There are neonatal vaccines under development and studies in Australia and in India have shown that exposure to neonatal rotavirus strains confers protection against severe diarrhea in future infections [36]. Phase I and II clinical trials of a neonatal vaccine being conducted in Australia have documented a $46 \%$ immune response in infants. This response has been associated with $56 \%$ efficacy against rotavirus infections among the same individuals the following winter [36]. A mathematical model that includes the application of a neonatal vaccine is presented in Section 5 .

\section{An extended model with vaccination}

The world health community believes that vaccines offer the best hope for protection against rotavirus infections [54]. Naturally acquired rotavirus infections provide protection against disease in a variety of ways including maternal antibodies. The search for a vaccine policy that will provide the most cost-effective way to stop rotavirus infections consequently, is of interest around the world [54]. The data from Australia exhibits strong seasonality trends and the possibility of "innovative" strategies such as the use of pulse vaccination perhaps should be considered (see $[\mathbf{4 1}])$.

The study led by Dr. Bishop showed that children who experienced neonatal rotavirus infections were significantly less likely to experience a severe rotavirus infections over the next three years [36]. Motivated by the potential efficacy of a neonatal vaccine, the model of Section 2 is modified to include a vaccinated class, $V(t)$ that includes all individuals who are vaccinated immediately after birth. It is assumed that the vaccine is applied only to the proportion $\lambda$ of non breast-fed 
newborns $((1-q) \mu N)$ per unit time and that the vaccine effectiveness wanes after an average period of $1 / \epsilon$. It is further assumed that infants who are neither breastfed nor vaccinated enter directly the susceptible class and that vaccination provides $100 \%$ protection until it wanes.

One could also consider another type of vaccine such as Rotarix (available in Mexico since July 2004). This vaccine will be given to infants at two months and four months of age. In order to account for this possibility in a rather crude way, we may consider vaccinating (with Rotarix) individuals from susceptible class at the rate $\phi$ (see Figure 3 ).

System (2.1) is modified as in Figure 3 to include two types of vaccination described above. The resulting model is given by the following system:

$$
\begin{aligned}
& M^{\prime}=q \mu N-\xi \beta M I / N-(\rho+\mu) M \\
& S^{\prime}=\mu[(1-\lambda)(1-q) N-S]-\beta S I / N-\phi S+\rho M+\delta R+\epsilon V \\
& L^{\prime}=\xi \beta M I / N+\beta S I / N-(\alpha+\mu) L \\
& I^{\prime}=\alpha L-(\gamma+\mu) I \\
& R^{\prime}=\gamma I-(\delta+\mu) R \\
& V^{\prime}=\lambda(1-q) \mu N+\phi S-(\epsilon+\mu) V
\end{aligned}
$$

where $N=M+S+L+I+R+V$ and $\beta=\beta_{0}\left[1+\beta_{1} \cos (2 \pi(t-\tau))\right]$.

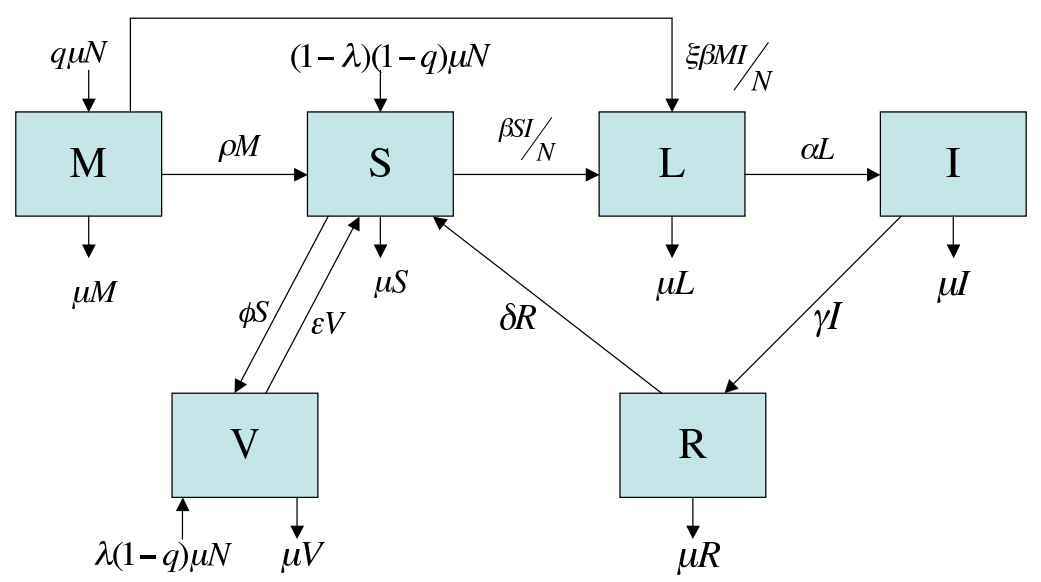

FIGURE 3. A schematic representation of the flow of individuals between epidemiological classes for System (5.1). 
First we consider the neonatal vaccine alone, that is, let $\phi=0$. Under the assumption of $\beta=\beta_{0}$, that is, $\beta_{1} \equiv 0$, the reproductive ratio under the controls $(\lambda$ and $q$ ) is given by:

$$
\Re(\lambda, q)=\frac{\alpha \beta_{0}[(1-q)(\epsilon+\mu(1-\lambda))(\rho+\mu)+q(\epsilon+\mu)(\rho+\xi \mu)]}{(\alpha+\mu)(\gamma+\mu)(\epsilon+\mu)(\rho+\mu)},
$$

that is,

$$
\Re_{0}:=\Re(0,0)=\frac{\beta_{0}}{\gamma+\mu} \times \frac{\alpha}{\alpha+\mu} .
$$

In order to see the potential impact of neonatal vaccination on reducing the prevalence of rotavirus infections, expression (5.2) is plotted as a function of $\lambda$ and $q$ (see Figure 4). Model (5.1) with $\phi=0$ has also been simulated as $\lambda$ varies using the base line parameter estimated in Section 3 (see Figure 5). Figure 4 shows that

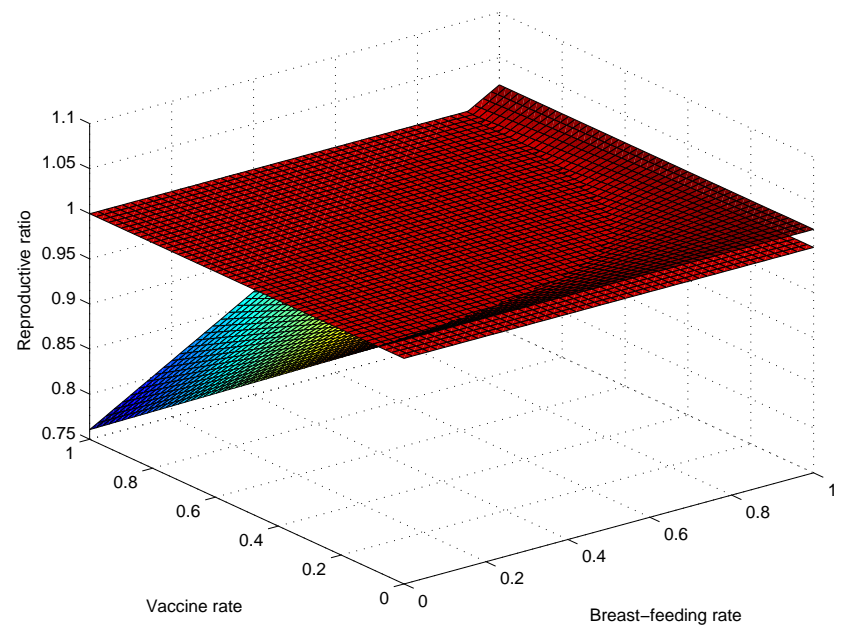

FiguRE 4. Reproductive ratio using neonatal vaccination rate $(\lambda)$ and breast-feeding rate $(q)$ using two months of breast-feeding duration. The place $\Re(\lambda, q)=1$ is shown for comparison.

the use of a neonatal vaccine and breast-feeding have stronger impact on reducing the reproductive ratio than the neonatal vaccine alone. Since breast-feeding rate in Australia (87.6\%) is already "high", applying a neonatal vaccine may be somewhat helpful.

We now consider a model for a vaccine that is given after birth (like Rotarix or RotaTeq [36]). The vaccine will be given to infants at two months and four months of age but this level of detail is not included in our model. In order to account for such vaccine policy in a rather crude way, we use Model (5.1) with $\lambda=0$. This model does not keep track of repeated vaccination but we suspect that the results will change minimally (see [8] for impact of re-vaccination using measles as an example). Nevertheless, we expect to explore this further but in the context of an age-structured model. 


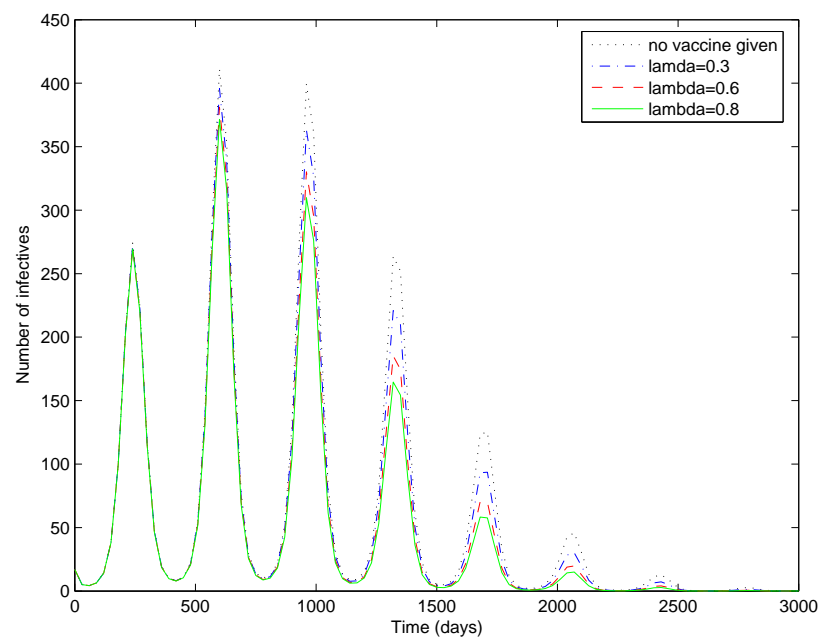

FIGURE 5. Simulated results with various neonatal vaccination regime $(\lambda=0,0.3,0.6,0.8)$. Parameters used: $\epsilon=0.0001, \alpha=0.5$, $\gamma=0.2 . \rho, \beta_{0}, \beta_{1}$ and $\delta$ are fixed at best-fit values from Table 1 .

The reproductive ratio under the control $(\phi$ and $q)$ is given by:

$$
\Re(\phi, q)=\frac{\alpha \beta_{0}[(\rho+\mu(1-q+\xi q))(\epsilon+\mu)+\xi \phi q \mu]}{(\alpha+\mu)(\gamma+\mu)(\epsilon+\phi+\mu)(\rho+\mu)} .
$$

In order to see the potential impact of vaccination on reducing the prevalence of rotavirus infections, expression (5.4) is plotted as $\phi$ and $\rho$ vary (see Figure 6). From Figure 6 one can note that this vaccine has a stronger impact (on reducing the basic reproductive ratio) than breast-feeding. The use of vaccine brings the possibility of $\Re(\phi, q)$ below one. In other words, a post breast-feeding vaccine seems more effective at controlling rotavirus disease than a neonatal vaccine (see Figure 4 and 6 ). Furthermore, the time required to bring down rotavirus disease from the population under consideration is shorter when using post breast-feeding than when using neonatal vaccines (Fig 5 and 7 ). The above results assume that the best-fit parameter values are the same as those that operated where a rotavirus infection was first introduced in Victoria, Australia. Of course, this is not the case in general. The values used correspond to an endemic rotavirus situation, that is, a situation where not all the individuals are susceptible. However, the fact that primary rotavirus infection occurs mostly on infants means that our estimates (or underestimates) are probably not too far off from the "real" values. Again, the main objective here is to explore various control scenarios on available data and not to make specific predictions. Consequently, our approach while not entirely rigorous illustrates the relative impact of various control measures on rotavirus transmission dynamics. 


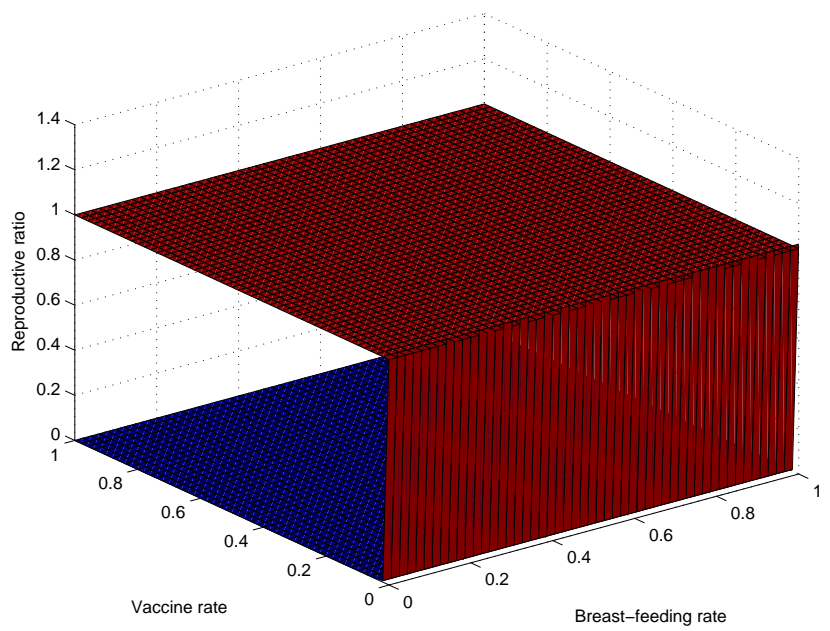

FiguRE 6. Reproductive ratio using post-breast feeding vaccination rate $(\phi)$ and breast-feeding rate $(q)$ using two months of breast-feeding duration. The place $\Re(\phi, q)=1$ is shown for comparison.

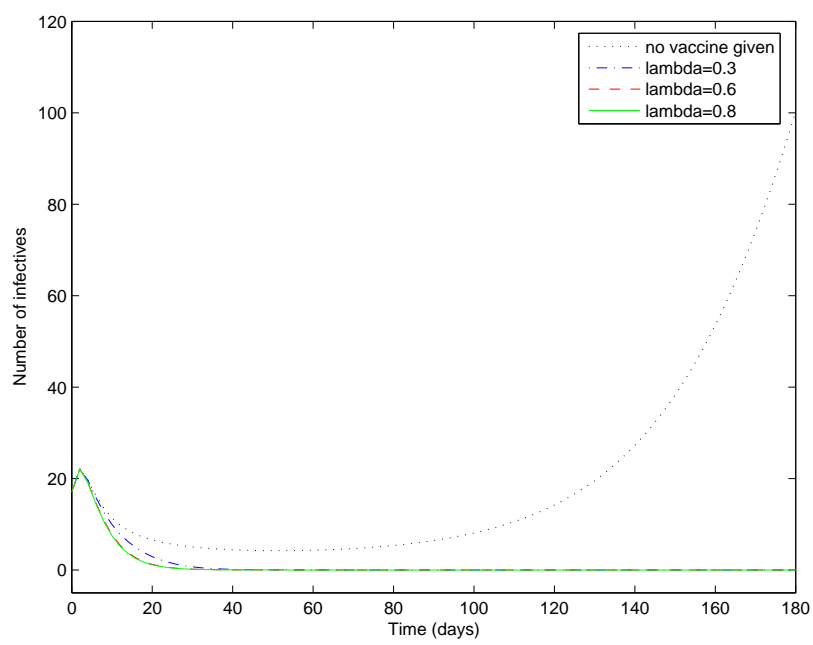

FiguRE 7. Simulated results with various after-birth vaccination rates $(\phi=0,0.3,0.6,0.8)$. Parameters used: $\epsilon=0.0001, \alpha=0.5$, $\gamma=0.2 . \rho, \beta_{0}, \beta_{1}$ and $\delta$ are fixed at best-fit values from Table 1 . 


\section{Age-structured model}

The high correlation between age and rotavirus incidence and prevalence is well known. In fact, since natural rotavirus infections confer protection against subsequent infections, adults are known to have lower prevalence of rotavirus infections. Furthermore, in general, each new infection tends to reduce the severity of diarrhea [45]. Mothers with antibodies due to prior infections protect their breast-fed infants against their first infections. The infection rate of infants younger than 6 months of age is much lower than that of those with ages in the 6 to 24 month range [26]. Early studies of rotavirus immunity have found that incomplete natural immunity is acquired from prior infections. Here, it is assumed that in general treated or recovered individuals can become re-infected while infants are assumed to be totally protected by maternal antibodies while they are being breast-fed. Writing a model with all these elements is possible and desirable particularly if the goal is to explore the heterogeneous impact that a rotavirus vaccine is likely to have or even the remote possibility of applying two distinct vaccines (neonatal and post-breast feeding vaccines) on the same population. There are additional issues and cost is one of them. Unfortunately, there is limited theoretical work on cost and epidemics. Here, a summary of some recent work on "optimal" vaccination strategies for agestructured populations is provided in order to bring this issue within the context of this volume [40].

In order to explain the approach an age-structured framework is introduced. The population is divided into six age-dependent classes: infants who breast-feed, susceptible, latent, infectious, recovered and vaccinated. $M(t, a), S(t, a), L(t, a)$, $I(t, a), J(t, a)$ and $R(t, a)$ denote their respective age densities. Using the parameters in Table 2, the following age-structured model can be formulated:

\begin{tabular}{|c||l|}
\hline$\Lambda$ & birth rate (assumed constant) \\
\hline$q$ & the proportion of infants who breast-feed (assumed constant) \\
\hline$\rho$ & $\begin{array}{l}\text { the constant per capita rate of departure from the breast-feeding } \\
\text { class into the susceptible class }\end{array}$ \\
\hline$\mu(a)$ & the age-specific per-capita natural death rate \\
\hline$\beta(a)$ & $\begin{array}{l}\text { the age-specific probability of becoming infected per contact } \\
\text { (with an infectious individuals) }\end{array}$ \\
\hline$c(a)$ & the age-specific per-capita contact/activity rate \\
\hline$\phi(a)$ & the per-capita rate at which susceptible individuals are vaccinated \\
\hline$\delta$ & the reductions in risk from prior exposure to a rotavirus $(0 \leq \sigma \leq 1)$ \\
\hline$\epsilon$ & the reductions in risk from a vaccine $(0 \leq \delta \leq 1)$ \\
\hline$\alpha$ & $\begin{array}{l}\text { the per-capita rate of progression from the latent } \\
\text { to the infectious class }\end{array}$ \\
\hline$\gamma$ & the per-capita recovery rate \\
\hline$p\left(t, a, a^{\prime}\right)$ & $\begin{array}{l}\text { the probability that an individual of age } a \text { has contact with an } \\
\text { individual of age } a^{\prime} \text { given that he has contact with a member } \\
\text { of population }\end{array}$ \\
\hline
\end{tabular}

TABLE 2. The definition of parameters 


$$
\begin{aligned}
\left(\frac{\partial}{\partial t}+\frac{\partial}{\partial a}\right) M(t, a) & =-\rho M(t, a)-\mu(a) M(t, a) \\
\left(\frac{\partial}{\partial t}+\frac{\partial}{\partial a}\right) S(t, a) & =\rho M(t, a)-\beta(a) c(a) B(t) S(t, a)-\phi(a) S(t, a)-\mu(a) S(t, a) \\
\left(\frac{\partial}{\partial t}+\frac{\partial}{\partial a}\right) V(t, a)= & \phi(a) S(t, a)-\epsilon \beta(a) c(a) B(t) V(t, a)-\mu(a) V(t, a) \\
\left(\frac{\partial}{\partial t}+\frac{\partial}{\partial a}\right) L(t, a)= & \beta(a) c(a) B(t)[S(t, a)+\delta R(t, a)+\epsilon V(t, a)] \\
\left(\frac{\partial}{\partial t}+\frac{\partial}{\partial a}\right) I(t, a)= & \alpha L(t, a)-[\gamma+\mu(a)] I(t, a) \\
\left(\frac{\partial}{\partial t}+\frac{\partial}{\partial a}\right) R(t, a) & =\gamma I(t, a)-\delta \beta(a) c(a) B(t) R(t, a)-\mu(a) R(t, a) \\
B(t) & =\int_{0}^{\infty} \frac{i\left(t, a^{\prime}\right)}{n\left(t, a^{\prime}\right)} p\left(t, a, a^{\prime}\right) d a^{\prime}
\end{aligned}
$$

where

$$
\begin{aligned}
& p\left(t, a, a^{\prime}\right)=\frac{c\left(a^{\prime}\right) n\left(t, a^{\prime}\right)}{\int_{0}^{\infty} c(u) n(t, u) d u}, \\
& c(a) n(a, t) p\left(a, a^{\prime}, t\right)=c\left(a^{\prime}\right) n\left(a^{\prime}, t\right) p\left(a^{\prime}, a, t\right), \\
& M(t, 0)=q \Lambda, \quad S(t, 0)=(1-q) \Lambda, \\
& V(t, 0)=L(t, 0)=I(t, 0)=J(t, 0)=0, \\
& M(0, a)=M_{0}(a), \quad S(0, a)=S_{0}(a), \quad V(0, a)=V_{0}(a), \\
& L(0, a)=L_{0}(a), \quad I(0, a)=I_{0}(a), \quad R(0, a)=R_{0}(a), \\
& n(t, a)=M(t, a)+S(t, a)+V(t, a)+L(t, a)+I(t, a)+R(t, a) .
\end{aligned}
$$

After some calculation that we do not present here (see [40] for more details) the reproductive number under vaccination policy $\phi(a)$, breast-feeding $q$, and average rate of antibody immunity loss $\rho$ is defined as $\Re(\phi, q, \rho)$ where

$$
\Re(\phi, q, \rho)=\int_{0}^{\infty} \int_{0}^{\infty} \frac{\alpha}{\gamma-\alpha} p_{\infty}(\tau+h)\left[e^{-\alpha \tau}-e^{-\gamma \tau}\right] \beta(h) c(h)\left[s^{*}(h)+\epsilon v^{*}(h)\right] d h d \tau,
$$

with $s^{*}(h)+\epsilon v^{*}(h)=q \Phi(0, h) \int_{0}^{h} \tau e^{-\tau \rho}[\Phi(0, \tau)]^{-1} d \tau+(1-q) \Phi(0, h)+\epsilon \int_{0}^{h} \phi(\tau) s^{*}(\tau) d \tau$ and $\Phi(a, b)=e^{-\int_{a}^{b} \phi(\tau) d \tau}$. The goal associated with the use of this model is that of finding the policy, that is, the triple $(\phi(a): a \in[o, \infty), q, \rho)$ that generates some "optimal" benefit. Here, we follow the work in $([\mathbf{7}],[\mathbf{1 9}],[\mathbf{3 8}])$ which implicitly assumes that reductions in prevalence are directly linked to reductions in $\Re(\phi, q, \rho)$. It is assumed that $q$ and $\rho$ are fixed.

The goal is to find the function $\phi$ that minimizes $\Re(\phi, q, \rho)$ in some sense. Following the approach described in $([\mathbf{1 9}])$ the functional $F(\phi, a, \rho)=\Re(0, q, \rho)-$ $\Re(\phi, q, \rho)$ is minimized under specific conditions. If one lets $C(\phi, q, \rho)$ denote the total cost associated with the implementation of vaccination strategy $(\phi, q, \rho)$ then under the assumption that such a cost function is a linear function of $\phi$, we formulate and solve the following optimization problems ( $q$ and $\rho$ are fixed) [40]: 
(I) Find a vaccination strategy $(\phi(a), q, \rho)$ that minimizes $C(\phi, q, \rho)$ under constraint that $\Re(\phi, q, \rho) \leq \Re_{*}$,

(II)Find a vaccination strategy $(\phi(a), q, \rho)$ that minimizes $\Re(\phi, q, \rho)$ under constraint that $C(\phi, q, \rho) \leq C_{*}$,

where $\Re_{*}$ and $C_{*}$ have pre-specified constant values. Using the transformation,

$$
\psi(a)=q \rho \phi(a) e^{-\int_{0}^{a} \phi(s) d s} \int_{0}^{a} e^{-\rho h} e^{\int_{0}^{h} \phi(s) d s} d h+(1-q) \phi(a) e^{-\int_{0}^{a} \phi(s) d s},
$$

the optimization problems become linear in $\psi([\mathbf{1 9}])$. The results that we obtained are $([\mathbf{7}],[\mathbf{2 0}],[\mathbf{4 0}])$ :

There are two possible optimal vaccination strategies for Problem (I).

(i) one-age strategy, that is, vaccinate the susceptible population at exactly age $\Omega$ or

(ii) two-age strategy, that is, vaccinate a fraction of the susceptible population at age $\Omega_{1}$ and a second fraction of susceptible at age $\Omega_{2}$ where $\Omega_{1}$ and $\Omega_{2}$ are functions of $q$ and $\rho$.

For a two-age strategy, $\Omega_{1}$ and $\Omega_{2}$ can be found by minimizing the expression $C\left(\Omega_{1}, \Omega_{2}\right)$ on $\Omega_{1} \in\left[0, \Omega_{*}\right]$ and $\Omega_{2} \in\left[\Omega_{*}, \infty\right)$, where

$$
C\left(\Omega_{1}, \Omega_{2}\right)=\frac{\omega-K\left(\Omega_{2}\right)}{K\left(\Omega_{1}\right)-K\left(\Omega_{2}\right)} Z\left(\Omega_{1}\right)+\frac{K\left(\Omega_{1}\right)-\omega}{K\left(\Omega_{1}\right)-K\left(\Omega_{2}\right)} Z\left(\Omega_{2}\right)
$$

which is the cost function of optimal ages $\Omega_{1}$ and $\Omega_{1}$. (See [40] for definitions of $\omega$ and $Z\left(\Omega_{i}\right), i=1,2$.) For problem (II), a similar result is obtained. In general, these conclusions state that the most cost-effective way of vaccinating a population is to have a program that vaccinates them at a discrete (small) number of age classes. The specific ages depend on the cost function.

\section{Conclusion}

The first rotavirus vaccine was withdrawn due to its sufficient correlation with intussusception in October 1999. Now we have two promising vaccines; Rotarix (by GlaxoSmithKline) available in Mexico City and Rotateq (by Merck) and awaiting approval of the Food and Drug Administration (FDA) for use in the U.S. Rotarix by GlaxoSmithKline gained its approval for use in Mexico in July 2004. It has already filed for market approval in more than 20 countries plus additional ones in Europe (in 2004) and there are plans to launch it in additional countries in Latin American and Asia. Merck hopes to get approval for its vaccine, RotaTeq in the U.S. In order to prove its safety, Merck conducted a rotavirus efficacy and safety trial (called REST) of RotaTeq on more than 70,000 children in the U.S. and in some European countries $([\mathbf{2 1}],[\mathbf{3 6}])$.

We now have advanced vaccines that are close to their implementations. However, several issues still need to be considered, particularly related to their implementation in poor countries. Vaccines efficacy trials must consider the "quality of maternal antibodies" which differ from population to population, country-specific breast-feeding rates, and the possibility of infection with multiple enteric viruses in (South African) infants [36]. In this paper we investigated the role of maternal antibodies on the dynamics of rotavirus infections and provided a platform that allows a rough comparison of the impact of two policies (vaccination and breast-feeding). In developing countries these issues are particulary important when polio enters in 
the equation. Dr. Timo Vesikari stated in the sixth international rotavirus symposium $[\mathbf{3 6}]$ that the oral polio vaccine (OPV) suppresses uptake of the rotavirus vaccine so co-administration of rotavirus vaccine with OPV needs to be avoided (given the fact that polio is still a serious threat, particularly in Africa).

Rotavirus exhibits a seasonal pattern. Thus the timing of vaccine can be critical and the use of pulse vaccination may hold some promise (see [41]). In the sixth International Rotavirus Symposium in Mexico Bishop noted that "widespread infections in newborns appears to reduce the prevalence of severe diarrhea in young children in the surrounding communities" [36]. In this paper we considered two possible vaccination strategies. Model (5.1) describes the role of neonatal vaccines (under development) which is based on strains of rotavirus that enter newborn nurseries in hospitals [36]. Using model (5.1) and simulations, we demonstrated the potential impact of neonatal vaccines and their effectiveness is compared to that of the maternal antibodies conferred by breast-feeding. Model (5.1) with $\lambda=0$ was used to study the potential impact of future vaccines such as RotaTeq and Rotarix. Seasonal patterns of rotavirus infections were considered. Different impacts of breast-feeding duration and vaccination rate on reproductive ratio were compared. Our studies also concluded that a regular (after-birth) vaccine is more effective in controlling rotavirus diseases than a neonatal vaccine.

Finally, it is clear that we can easily formulate models that accommodate two types of vaccines (neonatal and regular). Model results suggest that their joint use would be most effective in reducing rotavirus prevalence. However, whether or not such implementation is feasible on biological, risk or economic terms needs to be examined. The benefits of breast-feeding are substantial in multiple ways, hence post-breast feeding vaccines such as Rotarix and RotaTeq have an advantage in the sense that they can be applied to infants after breast-feeding so their impact can be combined.

With this work we hope to increase our understanding of rotavirus infections as the world proceeds with the implementation and development of rotavirus vaccines. The models proposed here can be expanded to incorporate multiple doses of oral vaccines and decreasing rates of subsequent infections. Making clear and accurate connections between geographic and seasonal patterns of rotavirus infections may be critical to our efforts to reduce disease burden.

\section{Acknowledgment}

The authors would like to thank Dr. Paul Kilgore in International Vaccine Institute and Dr. Robbin F. Itzler in Merck \& Co., Inc. for valuable discussions about the role of maternal antibodies and their waning effect.

\section{References}

[1] E. J. Anderson, S. G. Weber, Rotavirus infection in adults, Lancet Infect. Dis. 4(2) (2004), 91-99.

[2] H. T. Banks, H. K. Nguyen, Sensitivity of dynamical system to Banach space parameters, CRSC Tech Rep., CRSC-TR05-13, N.C. State University, Feb., 2005; J. Math. Anal. Appl. (to appear)

[3] R. Berger, F. Hadziselimovic, M. Just, F. Reigel, Influence of breast milk on nosocomial rotavirus infections in infants, Infection 12 (1984), 171-174.

[4] P. A. Blake, S. Ramos, K. L. Mac Donald, et al, Pathogen specific risk factors and protective factors for acute diarrheal disease in urban Brasilian infants, J. Infect. Dis. 167 (1993), 627-632. 
[5] F. Brauer, The Kermack-McKendrick epidemic model revisited, Math. Biosci. (2005) (to appear)

[6] J. B. Carlin, P. Chondros, P. Masendycz, H. Bugg, R. F. Bishop, G. L. Barnes, Rotavirus infection and rates of hospitalisation for acute gastroenteritis in young children in Australia, 1993-1996, Medical J. of Australia 168 (1998), 252-256.

[7] C. Castillo-Chavez, Z. Feng, Global stability of an age-structure model for TB and its applications to optimal vaccination strategies, Math. Biosc. 151 (2) (1998), 135-154.

[8] C. W. Castillo-Chavez, J. C. Villarreal Aranda, Three models for Measles Control, MTBI Technical reports, 1997.

[9] I. L. Chrystie, B. M. Totterdell, J. E. Banatvala, Asymptomatic endemic rotavirus infections in the newborn, Lancet 1 (1978), 1176-1178.

[10] H. F. Clark, P. A. Offit, Vaccines for rotavirus gastroenteritis universally needed for infants, Pediatr. Ann. 33 (8) (2004), 536-543.

[11] J. Clemens, M. Rao, F. Ahmed, et al, Breast-feeding and the risk of life-threatening rotavirus diarrhea prevention or postponement?, Pediatrics. 92 (1993), 680-685.

[12] S. M. Cook, R. I. Glass, C. W. LeBaron, M. S. Ho, Global seasonality of rotavirus infections, Bull. World Health Organ. 68 (1990), 171-177.

[13] N. A. Cunliffe, J. S. Bresee, C. A. Hart, Rotavirus vaccines: development, current issues and future prospects, J. Infect. 45 (1) (2002), 1-9.

[14] A. S. Cunningham, Breast-feeding and health, J. Pediatr. 110 (1987), 658-659.

[15] M. Davidian, D. M. Giltinan, Nonlinear Models for Repeated Measurement Data, Chapman and Hall/ CRC, Boca Raton, 1995.

[16] L. C. Duffy, M. Riepenhoff-Talty, T. E. Byers, et al, Modulation of rotavirus enteritis during breast-feeding: Implications on alterations in the intestinal bacterial flora, Am. J. Dis. Child. 140 (1986), 1164-1168.

[17] A. R. Gallant, Nonlinear Statistical Models. Wiley Series in Probability and Mathematical Statistics: Applied Probability and Statistics, John Wiley \& Sons, Inc., New York, 1987.

[18] M. Gurwith, W. Weirman, D. Hinde, et al, A prospective study of rotavirus infection in infants and young children, J. Infect. Dis. 114 (1981), 218-224.

[19] K. P. Hadeler, J. Müller, Optimal harvesting and optimal vaccination In submission

[20] K. P. Hadeler, J. Müller, Vaccination in age-structured populations II: Optimal vaccination strategies, In V. Isham and G. Medley, editors, Models for Infectious Human Diseases: Their Structure and Relation to Data, Cambridge University Press, (1993), 102-114.

[21] P. M. Heaton, M. G. Goveia, J. M. Miller, P. Offit, H. F. Clark, Development of a pentavalent rotavirus vaccine against prevalent serotypes of rotavirus gastroenteritis, J. Infect. Dis. 192 (2005), Suppl 1:S17-21.

[22] P. W. Howie, J. S. Forsyth, S. A. Ogston, A. Clark, C. D. Florey, Protective effect of breastfeeding against infection, Br. Med. J. 300 (1990), 11-16.

[23] R. I. Jennrich, Asymptotic properties of non-linear least squares estimators, Ann. Math. Statist. 40 (1969), 633-643.

[24] B. Jiang, J. R. Gentsch, R. I. Glass, The role of serum antibodies in the protection against rotavirus disease: an overview, Clin. Infect. Dis. 34 (2002), 1351-1361.

[25] A. Z. Kapikian, A rotavirus vaccine for prevention of severe diarrhoea of infants and young children: development, utilization and withdrawal, Novartis Found Symp. 238 (2001), 153171; discussion, 171-179.

[26] A. Z. Kapikian, H. W. Kim, R. G. Wyatt, W. L. Cline, J. O. Arrobio, C. D. Brandt, W. J. Rodriguez, D. A. Sack, R. M. Chanock, R. H. Parrott, Human reovirus-like agent as the major pathogen associated with "winter" gastroenteritis in hospitalized infants and young children, N. Engl. J. Med. 294 (1976), 965-972.

[27] L. Mata, A. Simhon, J. J. Urrutia, R. A. Kronmal, R. Fernandez, B. Garcia, Epidemiology of rotaviruses in a cohort of 45 Guatamalan Mayan Indian children observed from birth to the age of three years, J. Infect. Dis. 148(3) (1983), 452-461.

[28] A. B. Naficy, R. Abu-Elyazeed, J. L. Holmes, et al, Epidemiology of rotavirus diarrhea in Egyptian children and implications for disease control, Am. J. Epidemiol. 150 (1999) 770-777.

[29] D. S. Newburg, J. A. Peterson, G. M. Ruiz-Palacios, et al, Role of human-milk lactadherin in protection against symptomatic rotavirus infection, Lancet. 351 (1998) 1815-1816.

[30] M. Nuno, G. Chowell, X. Wang, C. Castillo-Chavez, On the role of cross-immunity, vaccines and "flu" survival, (submitted) 
[31] E. Mastretta, P. Longo, A. Laccisaglia, L. Balbo, R. Russo, A. Mazzaccara, P. Gianino, Effect of Lactobacillus $G G$ and breast-feeding in the prevention of rotavirus nosocomial infection, J. Pediatr. Gastroenterol. Nutr. 35(4) (2002), 527-531.

[32] T. V. Murphy, M. G. Paul, S. M. Mehran, D. B. Nelson, A. O. Jumaan, C. A. Okoro, L. R. Zanardi, S. Setia, E. Fair, C. W. LeBaron, M. Wharton, J. R. Livingood; The Rotavirus Intussusception Investigation Team, Intussusception among Infants Given an Oral Rotavirus Vaccine, New Engl. J. Med. 344(8) (2001), 564-572.

[33] Pan American Health Organization, Family and Community Health Area, Immunization Unit. Regional Meeting on the Implementation of Rotavirus Epidemiological Surveillance: generating information for decision-making. Washington, D.C.: PAHO; 2003.

[34] U. D. Parashar, J. S. Bresee, J. R. Gentsch, and R. I. Glass, Rotavirus, Emerging infectious diseases 4(4) (1998), 561-570.

[35] U. D. Parashar, R. C. Holman, M. J. Clarke, J. S. Bresee, R. I. Glass, Hospitalizations associated with rotavirus diarrhea in the United States, 1993 through 1995: surveillance based on the new ICD-9-CM rotavirus specific diagnostic code J. Infect. Dis. 177 (1998), 7-13.

[36] Proceedings of the Sixth International Rotavirus Symposium, July 7-9, 2004, Mexico City.

[37] L. Roberts, Vaccines: Rotavirus vaccines' second chance, Science. 305(5692) (2004), 18901893.

[38] C. Rorres, W. Fair, Optimal harvesting policy for an age-specific population, Math. Biosci. 24 (1975), 31-47.

[39] G. A. F. Seber and C. J. Wild, Nonlinear Regression, John Wiley \& Sons, Inc., New York, 1989.

[40] E. Shim, Z. Feng, M. Martcheva, C. Castillo-Chavez, Age-structure model of rotavirus infection with vaccination, (submitted to J. Math. Biol.)

[41] B. Shulgin, L. Stone, Z. Agur, Pulse vaccination strategy in the SIR epidemic model, Bull. Math. Biol. 60(6) (1998), 1123-1148.

[42] T. J. Török, P. E. Kilgore, M. J. Clarke, R. C. Holman, J. S. Bresee, R. I. Glass, Visualizing geographic and temporal trends in rotavirus activity in the United States, 1991 to 1996, Pediatr. Infect. Dis. J. 16(10) (1997), 941-946.

[43] B. M. Totterdell, I. L. Chrystie, J. E. Banatvala, Rotavirus infections in a maternity unit, Arch. Dis. Child. 51 (1976), 924-928.

[44] A. W. Tucker, A. C. Haddix, J. S. Bresee, R. C. Holman, U. D. Parashar, R. I. Glass, Costeffectiveness analysis of a rotavirus immunization program for the United States, JAMA 279 (1998), 1371-1376.

[45] F. R. Velazquez, D. O. Matson, J. J. Calva, L. Guerrero, A. L. Morrow, S. Carter-Campbell, R. I. Glass, M. K. Estes, L. K. Pickering, G. M. Ruiz-Palacios, Rotavirus infections in infants as protection against subsequent infections, N. Engl. J. Med. 335(14) (1996), 1022-1028.

[46] T. Verstraeten, et al. Burden of Rotavirus in the new European Union. Poster presented at ESPID annual meeting, May 2005

[47] T. Vesikari, A. Karvonen, T. Korhonen, M. Espo, E. Lebacq, J. Forster, F. Zepp, A. Delem, B. De Vos, Safety and immunogenicity of RIX4414 live attenuated human rotavirus vaccine in adults, toddlers and previously uninfected infants, Vaccine 22(21-22) (2004), 2836-2842.

[48] T. Vesikari, M. ORyan, H. Abate, et al., Overcoming the safety hurdle: The rotavirus vaccine RIX4414 is not associated with intussusception, Oral presentation at ESPID annual meeting, May 2005, Valencia, Spain

[49] R. L. Ward, D. I. Bernstein, V. E. Smith, D. S. Sander, A. Shaw, J. J. Eiden, P. Heaton, P. A. Offit, H. F. Clark, Rotavirus immunoglobulin a responses stimulated by each of 3 doses of a quadrivalent human/bovine reassortant rotavirus vaccine, J. Infect. Dis. 189(12) (2004), $2290-2293$.

[50] http://www.gsk-bio.com/webapp/PressCorner/PressDetail.jsp?PressId=10399

[51] http://www.cdc.gov/nip/publications/fs/rotavirus.htm

[52] http://www.gsk.com/ControllerServlet?appId=4\&pageId=402\&newsid $=421$

[53] http://www.kellymom.com/writings/bf-numbers.html

[54] http://www.rotavirusvaccine.org/vaccine-facts.htm

[55] State of the art of new vaccines: research and development. As found at http://www.who.int/vaccine_research/documents/new_vaccines/en/ 
Department of Mathematics and Statistics, Arizona State University, P.O. Box 871804, TEMPE, AZ 85287-1804, USA

E-mail address: alicia@mathpost.asu.edu

Center for Research in Scientific Computation, North Carolina State University, P.O. Box 8205 Raleigh, NC 27695-8205, USA.

E-mail address: htbanks@unity.ncsu.edu

Department of Mathematics and Statistics, Arizona State University, P.O. Box 871804, TEMPE, AZ 85287-1804, USA.

E-mail address: chavez@math.asu.edu 